\title{
Essais d'embouche intensive de zebus maliens en Côte d'Ivoire
}

\author{
par J. L. JOUVE (*) et L. LETENNEUR (*) \\ RESUME
}

Des zébus maliens entretenus en alimentation intensive à base de Stylosanthes gracilis et de farines basses de riz n'ont eu qu'un gain de poids journalier moyen médiocre pour un indice de consommation très élevé.

Dans une autre expérience avec des animaux mieux choisis et disposant de Panicum maximum comme fourrage, des résultats nettement supérieurs ont été obtenus, mais toujours trop faibles pour justifier Ia vulgarisation de cette technique avec de tels animaux.

\section{A. PREMIERE EXPERIENCE}

Elle utilise des mâles castrés, âgés de 6 à 9 ans, d'un poids moyen de $331 \mathrm{~kg}$ et a pour objectif essentiel :

- d'étudier le comportement des zébus maliens de cet âge en stabulation libre et de mettre en évidence leurs possibles aptitudes à l'engraissement intensif, tout en permettant d'obtenir des informations précises sur la valeur des indices de consommation. Compte tenu de ces seuls objectifs, aucun lot témoin n'a été constitué.

\section{MATERIEL ET METHODES}

L'expérience a eu lieu de juillet à novembre, au Centre de Recherches Zootechniques de Bouaké-Minankro, en moyenne Côte d'Ivoire, dont le climat est très représentatif du type Baoulé-Dahoméen.

\section{Aménagements}

Les animaux sont entretenus en stabulation

(*) I.E.M.V.T., Centre de Recherches Zootechniques de Minankro, B.P. no 1152, Bouake, Côte d'Ivoire. libre dans un parc de $12 \times 10 \mathrm{~m}$, ce qui correspond à une aire de $10 \mathrm{~m}^{2}$ par animal. Une aire de $3 \times 12 \mathrm{~m}$ est couverte par des tôles d'aluminium. Elle abrite les mangeoires et permet aux animaux d'être protégés des intempéries. Le parc, relié à un couloir de contention, utilisé pour les différentes manipulations et les pesées, est pourvu d'un abreuvoir à remplissage manuel, muni d'un compteur d'eau.

\section{Animaux}

Ce sont des zébus importés du Mali et achetés sur le marché à bétail de Bouaké. Les 10 animaux, très hétérogènes, ont été vaccinés à leur entrée en Côte d'Ivoire contre la peste bovine et la péripneumonie. Au moment de leur mise en parc, ils subissent un traitement au Bérénil à titre de prévention contre la trypanosomose et reçoivent du tartrate de pyrantel contre leurs parasites gastro-intestinaux.

\section{Alimentation}

Leur alimentation est uniquement composée de Stylosanthes gracilis, dont Ia composition bromatologique moyenne est la suivante: 


\section{Matières sèches}

p. 100

20,29

p. 100

de

M.S.

Matières minérales

8,57

Matières azotées totales .

13,50

35,97

Matières cellulosiques

U.F. par kg de matière verte .

Matières azotées digestibles $\mathrm{g} / \mathrm{kg} \mathrm{M}$.V.

0,10

19,0

... et de la farine basse de riz, ainsi composée :

Matières sèches .

p. 100

85,77

p. 100

de

Matières minérales

M.S.

Matières cellulosiques . . . . . $\quad 9,39$

Matières grasses . . . . . . 17,46

Extractif non azoté . . . . . 47,09

U.F. par $\mathrm{kg}$ produit frais . . . . 0,94

M.A.D. $\mathrm{g}$.
Matières azotées totales . . . . . 16,62

- poids du gras de rognon;

- mesure de la longueur de la carcasse;

- mesure de l'épaisseur de la cuisse;

- mesure de l'épaisseur des muscles du plat de côtes.

Le poids de la carcasse est reconstitué par addition des poids des quatre quartiers, des rognons et du gras de rognon. La conformation de la carcasse et l'état d'engraissement font l'objet d'un jugement à l'œil. Les rendements calculés sont les suivants :

- Rendement sur poids vif :

$\mathrm{R}=\frac{\text { Poids de la carcasse pantelante } \times 100}{\text { Poids vif }}$

- Rendement vrai :

$\mathrm{R}=\frac{\text { Poids de la carcasse pantelante } \times 100}{\text { Poids vif }- \text { poids du contenu digestif }}$

Les abattages sont effectués à Bouaké. Les quartiers avant sont séparés des quartiers arrière par section entre la $7^{\mathrm{e}}$ et la $8^{\mathrm{e}}$ côte.

\section{RESULTATS}

L'expérience a duré 4 mois et demi. Les éléments de la ration, donnés ad libitum, sont distribués en quantités telles qu'il $y$ ait au moins 10 p. 100 de refus. Les aliments sont laissés en permanence à la disposition des animaux. La ration alimentaire est pesée au moment de sa distribution et les refus tous les matins. La consommation d'eau est contrôlée par le relevé du compteur. Les animaux sont pesés toutes les semaines, le matin à jeûn. Tous les mois ils passent sur la bascule trois jours de suite. Le poids retenu est la moyenne des trois opérations. Cette triple pesée permet de limiter les erreurs d'observation et sert de référence. L'état sanitaire des animaux est contrôlé quotidiennement. Des frottis sanguins sont pratiqués en cours d'expérience.

Les animaux sont abattus en fin d'expérience, après une période de jeûne de $24 \mathrm{~h}$ et les observations suivantes sont alors effectuées :

- poids vif au début de la période de jeûne;

— poids vif après $24 \mathrm{~h}$ de jeûne;

- poids des quatre quartiers;

- poids du Ve quartier (tête, peau, pied, queue et viscères);

- poids des rognons;

\section{Remarques générales}

a) Les animaux achetés et mis en parc le 12 juillet 1968 sont maigres.

Ils sont marqués le 15 et reçoivent tous le même traitement : Bérénil et Tartrate de pyrantel (Exhelm) le 20 juillet. Fortement infestés d'ixodes à leur arrivée, les animaux sont douchés avec une solution de Lindane le 13 juillet. Un douchage hebdomadaire permet ensuite un contrôle efficace des ectoparasites.

b) Ils s'habituent très rapidement à ce mode d'entretien, entièrement nouveau pour eux, et restent calmes tout au long de l'expérience.

Au cours de celle-ci, ils manifestent un comportement alimentaire très individualisé;

Il n'y a pas de bousculade au moment de la distribution des aliments, chacun venant boire ou manger aux moments qui lui conviennent.

c) L'un deux, qui a présenté une boiterie d'origine rhumatismale du postérieur droit à partir du début novembre, provoquant un amaigrissement important en fin d'essai, est exclu de l'évolution des poids. 
Evolution pondérale

La croissance journalière moyenne pour l'en- semble de l'expérience est de $298 \mathrm{~g}$. Cela représente une variation absolue moyenne de $41 \mathrm{~kg}$.

TABLEAU N ${ }^{\circ} \mathrm{I}$

Poids moyens et poids pondérés

\begin{tabular}{|c|c|c|c|c|c|c|c|c|c|}
\hline $\mathrm{D}$ a $\mathrm{t}$ e $\mathrm{s}$ & 12.7 .68 & 15.7 & 22.7 & 12.8 & 2.9 & 23.9 & 14.10 & 21.11 & 25.11 \\
\hline $\mathrm{N}$ & 9 & 9 & 9 & 9 & 9 & 9 & 9 & 9 & 9 \\
\hline $\begin{array}{l}\text { Poids } \\
\text { moyen (kg) }\end{array}$ & $\begin{array}{r}332,4 \\
+\quad 9,8\end{array}$ & $\begin{array}{l}324,3 \\
+11,1\end{array}$ & $\begin{array}{l}334,2 \\
+13,4\end{array}$ & $\begin{array}{r}337,6 \\
+15,5\end{array}$ & $\begin{array}{l}345,4 \\
+15,0\end{array}$ & $\begin{array}{l}351,2 \\
+16,6\end{array}$ & $\begin{array}{l}362,6 \\
+16,7\end{array}$ & $\begin{array}{r}369,6 \\
+14,0\end{array}$ & $\begin{array}{l}373,0 \\
+13,8\end{array}$ \\
\hline $\begin{array}{l}\text { Poids } \\
\text { pondété }\end{array}$ & & 100 & 103,0 & 104,1 & 106,5 & 108,2 & 111,8 & 113,9 & 115,0 \\
\hline
\end{tabular}

\section{Les consommations}

Ont été les suivantes :

a) Stylosanthes: le niveau de consommation a rapidement augmenté et s'est stabilisé à partir de la $3^{\mathrm{e}}$ semaine aux environs de $16 \mathrm{~kg}$ par jour et par animal. Cela représente une consommation journalière moyenne de $3,2 \mathrm{~kg}$ de matière sèche et 1,57 UF par animal. b) Farines de riz: les quantités de farine de riz consommées sont passées de $0,27 \mathrm{~kg}$ par animal et par jour la première semaine à plus de $5 \mathrm{~kg}$ par animal et par jour la quinzième semaine. Par la suite, le niveau de consommation s'est stabilisé aux environs de $5,3 \mathrm{~kg}$ par jour et par animal.

TABLEAU $\mathrm{N}^{\circ} \mathrm{II}$

Consommation

\begin{tabular}{|l|l|c|c|}
\hline & & Matières sèches & U.F. \\
\hline $\begin{array}{l}\text { Total pour le lot, } \\
\text { en } 136 \text { jours }\end{array}$ & $\begin{array}{l}\text { Stytosanthes } \\
\text { Farines de riz } \\
\text { Total }\end{array}$ & $\begin{array}{l}4341 \mathrm{~kg} \\
5097 \mathrm{~kg}\end{array}$ & $\begin{array}{l}139 \\
9438 \mathrm{~kg}\end{array}$ \\
\hline $\begin{array}{l}\text { Moyenne par animal } \\
\text { et par jour }\end{array}$ & & $\frac{596}{725}$ & $\frac{552}{958} \mathrm{~kg}$ \\
\hline
\end{tabular}

c) Abreuvement : la consommation d'eau est passée de 7,7 litres par jour et par animal à environ 25 litres à la $8^{\mathrm{e}}$ semaine. Elle s'est maintenue à ce niveau par la suite, et la moyenne journalière pour la durée de l'expérience a été de 22,5 litres.

d) Indices de consommation : varie selon les périodes. Il est très élevé et toujours supérieur à 10. En fin d'essai, la valeur de l'indice cumulé atteint 19.

TAHLEAU $\mathrm{N}^{\circ}$ III

Gains de poids

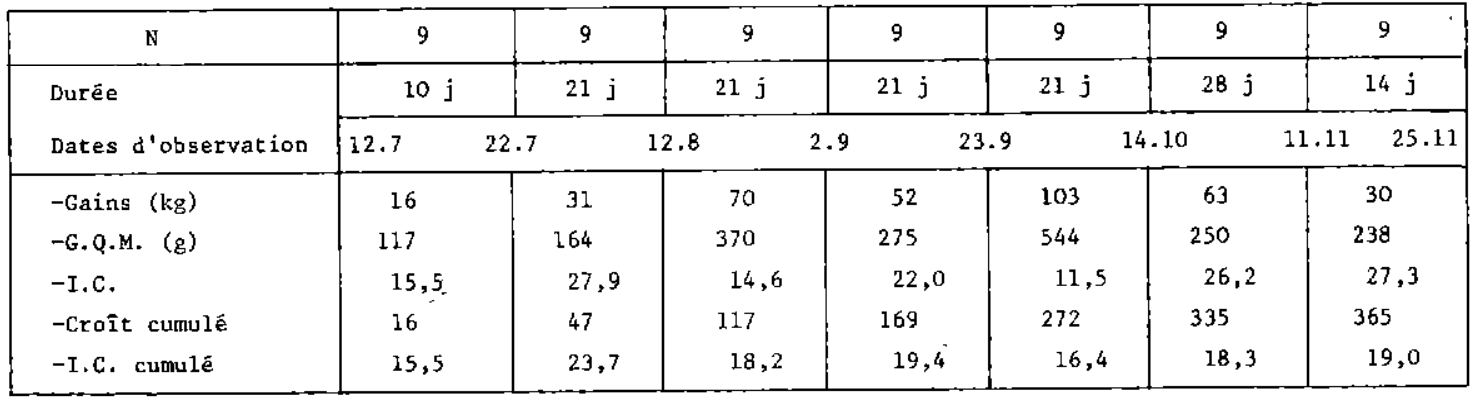




\section{Etude de carcasses}

Les animaux ont été abattus en deux lots de cinq animaux : le premier lot le 27 novembre, le second le 10 décembre. Les résultats figurent dans le tableau IV. La conformation bouchère est bonne, à profils musculaires rectilignes à sub-convexes.

L'état d'engraissement est bon, gras de rognon et graisse interne importants. La graisse musculaire et la graisse de couverture existent en quantité limitée.

Etude des carcasses

\begin{tabular}{|c|c|}
\hline & Moyen $\mathrm{n}$ e $\mathrm{s}$ \\
\hline $\begin{array}{l}\text { Poids vif avant jeûne } \\
\text { Poids vif après } 24 \mathrm{~h} \text { de jeûne } \\
\text { Poids du contenu digestif } \\
\text { Poids vif vide } \\
\text { Poids carcasse } \\
\text { Rendement sur poids vif } \\
\text { Rendement sur poids vif après } 24 \mathrm{~h} \\
\text { Rendement sur poids vide }\end{array}$ & $\begin{array}{r}371,7 \\
358,4 \\
43,7 \\
314,7 \\
199,3 \\
53,61 \\
55,60 \\
63,33\end{array}$ \\
\hline $\begin{array}{l}\text { Poids carcasse chaude } \\
\text { Poids arriëres } \\
\text { Poids arrières en p.100 carcasse } \\
\text { Poids avants } \\
\text { Poids avants p.100 carcasse } \\
\text { Poids gros rognon } \\
\text { Poids gros rognon p.100 carcasse }\end{array}$ & $\begin{array}{r}199,3 \\
111,9 \\
56,1 \\
87,4 \\
43,9 \\
3,9 \\
1,95\end{array}$ \\
\hline $\begin{array}{l}\text { Longueur de carcasse } \\
\text { Indice de compacité } \\
\text { Epaisseur de la cuisse } \\
\text { Epaisseur cuisse p.100 carcasse } \\
\text { Epaisseur cuisse p.100 L } \\
\text { Epaisseur plat de côte }\end{array}$ & $\begin{array}{r}120,3 \\
1,65 \\
23,2 \\
11,6 \\
19,3 \\
2,6\end{array}$ \\
\hline
\end{tabular}

\section{CONCLUSION}

Cette première expérience d'embouche intensive, réalisée en Côte d'Ivoire, a pour objectif immédiat de définir l'aptitude à l'engraissement intensif des zébus maliens importés et présentés maigres sur les marchés à bestiaux des grands centres.

Le fourrage distribué est du Stylosanthes gracilis, légumineuse fourragère qui donne, en Côte d'Ivoire, des rendements élevés à l'hectare. L'aliment concentré consiste en des farines basses de riz, sous-produit industriel disponible en quantités importantes.

10 animaux ont été soumis à cette expérience qui a duré quatre mois et demi, du 12 juillet au 27 novembre 1968.

Les résultats généraux obtenus sont les suivants : $\mathrm{kg}$

par animal

— poids moyen au départ - . 332,4

— poids en fin d'expérience . . 373,0

- gain de poids moyen . . . 40,6

ce qui représente un gain journalier de $298 \mathrm{~g}$ par jour, par animal, pendant 136 jours.

Pour l'ensemble de la période d'engraissement, l'indice de consommation a été de 19 UF.

Cette expérience a surtout une valeur d'orientation. Les renseignement obtenus sur les possibilités de crôt et les indices de consommations sont cependant trop partiels pour que des conclusions vraiment valables puissent en être tirées.

Ils doivent être complétés par d'autres expériences qui permettront, en outre, d'étudier l'économie de cette modalité d'embouche intensive de zébus maliens en Côte d'Ivoire. 


\section{B. DEUXIEME EXPERIENCE}

Elle porte sur des mâles castrés, âgés de 3 à 5 ans, d'un poids moyen au départ de $231 \mathrm{~kg}$.

Complémentaire de la précédente, puisqu'elle s'adresse à des animaux de même race et de même origine mais plus jeunes, cette expérience se déroule suivant un protocole identique tout en utilisant le même type d'alimentation concentrée, le Stylosanthes étant par contre remplacé par du Panicum maximum.

Elle a essentiellement eu pour objectif d'étudier les possibilités d'engraissement de ce type d'animaux, de préciser la valeur des indices de consommation afin de fixer le prix de revient du kg ajouté, et d'établir ainsi le seuil de rentabilité d'une telle opération en Côte d'Ivoire.

\section{MATERIEL ET METHODES}

L'expérience a duré du 16 mai 1970 au 20 septembre de la même année, soit 130 jours.

Les animaux choisis, au nombre de 15 , sont d'un format moyen et maigres. Deux d'entre eux sont tirés au sort pour être immédiatement abattus à titre de témoins. Leurs carcasses sont l'objet d'observations et de mesures devant servir d'éléments de référence et de comparaison pour l'abattage final.

Les autres sont mis en stabulation dans le parc du C.R.Z. dans les mêmes conditions que précédemment.

Dès leur mise en parc ils ont reçu :

- une injection d'anthrycide prosalt destinée à les prémunir contre les trypanosomoses; une injection choc de vitamines comprenant: vitamine $\mathrm{A}-2.000 .000 \mathrm{UI}$; vitamine $\mathrm{D}_{3}$ 300.000 UI; vitamine E-200 UI et une administration orale de tartrate de pyrantel (Exhelm) contre les parasites gastro-intestinaux.

\section{Alimentation}

L'aliment concentré, farine basse de riz, est le même alors que du Panicum maximum, produit en parcelle irriguée, est substitué au Stylosanthes gracilis.

Les analyses de laboratoire permettent d'attribuer aux éléments de la ration les valeurs alimentaires moyennes suivantes:

\begin{tabular}{|l|c|c|c|c|c|}
\hline Désignation & M.S. & U.F. & M.A.D. & Ca & P \\
\hline Panicum maximum par kg & $200 \mathrm{~g}$ & 0,11 & $14 \mathrm{~g}$ & $1,2 \mathrm{~g}$ & $0,3 \mathrm{~g}$ \\
\hline Farines de riz par kg & $850 \mathrm{~g}$ & 0,85 & $95 \mathrm{~g}$ & $0,5 \mathrm{~g}$ & $12,0 \mathrm{~g}$ \\
\hline
\end{tabular}

\section{Conduite de l'expérience}

Comme pour l'expérience précédente, les aliments sont donnés à volonté et les quantités distribuées sont telles qu'il y ait au moins $10 \mathrm{p}$. 100 de refus.

Les observations sont relatives au contrôle des consommations, aux mesures de croissance, à la surveillance de l'état sanitaire et à l'étude des carcasses.

L'aliment vert est distribué deux fois, le matin et le soir; les quantités données sont pesées régulièrement. L'aliment concentré est pesé et distribué le matin, après enlèvement des refus de la veille.
Les refus sont pesés chaque matin; les quantités d'eau relevées quotidiennement sur les compteurs.

Les animaux sont pesés individuellement chaque semaine et cinq triples pesées de référence ont eu lieu aux dates suivantes : $16 \mathrm{mai}$, 12 juin, 16 juillet, 28 août, 22 septembre.

\section{Etat sanitaire}

L'état sanitaire est l'objet d'une surveillance quotidienne. La suspicion de cas de trypanosomose nous a conduit à renouveler par deux fois le traitement à l'anthrycide sur l'ensemble du lot. 
Etude des carcasses.

Les animaux ont été abattus à Abidjan le 23 septembre 1970, après $24 \mathrm{~h}$ de jeûne et seules les observations suivantes ont été relevées :

- poids vif au départ de Bouaké;

- poids de la carcasse chaude;

- mesure de la longueur de carcasse;

- mesure de l'épaisseur de la cuisse;

- mesure de l'épaisseur des muscles du plat de côte.

Le rendement calculé est le rendement sur poids vif, évalué de la façon suivante :

$\mathbf{R}=\frac{\text { Poids de la carcasse pantelante } \times 100}{\text { Poids vif }}$

\section{RESULTATS}

Les animaux se sont rapidement habitués à des conditions d'entretien totalement nouvelles pour eux. Toutefois, plusieurs facteurs d'ordre divers sont intervenus en cours d'expérience pour en perturber le déroulement. Il nous semble important de les étudier avant l'exposé des rêsultats :

- La litière mise à la disposition des animaux au début de l'expérience consistait en de la paille de Stylosanthes. Trop ligneuse, elle a fini par provoquer des blessures podales dont la sanction fut une perte de poids pour l'ensemble du lot. Cette litière fut remplacée par de la paille d'Imperata qui donna par la suite toute satisfaction.

- après un mois de séjour en parc, deux animaux ont dû être éliminés; l'un était atteint de streptothricose suppurée affectant gravement son état général; l'autre n'avait cessé de perdre du poids depuis le début de l'essai, sans raison apparente.

Le lot expérimental s'est donc trouvé réduit à onze têtes et les résultats sont rapportés à cet effectif.

\section{Evolution du poids}

Le gain de poids moyen est de $420 \mathrm{~g}$ par animal et par jour pour toute la durée de l'expérience.

TABLEAU $\mathrm{N}^{\circ} \mathrm{I}$

Poids moyens et poids pondérés

\begin{tabular}{|c|c|c|c|c|c|}
\hline Durëe & & & $35 \mathrm{j}$ & $42 j$ & $26 \mathrm{j}$ \\
\hline Eates & 16.5 & 12.6 & 16.7 & 28.8 & 22.9 \\
\hline $\mathbf{N}$ & 11 & 11 & 11 & 11 & 11 \\
\hline Poids moyen & $\begin{array}{r}228,9 \\
\pm \quad 15,3\end{array}$ & $\begin{array}{r}236,9 \\
\pm 16,5\end{array}$ & $\begin{array}{r}250,18 \\
\pm 15,4\end{array}$ & $\begin{array}{r}273,18 \\
+\quad 19,3\end{array}$ & $\begin{array}{r}283,45 \\
\pm 18.7\end{array}$ \\
\hline Poids pondéré & 100 & 103,5 & 109,2 & 119,3 & 123,8 \\
\hline
\end{tabular}

TABLEAU $\mathrm{N}^{\circ} \mathrm{II}$

Gaing de poids

\begin{tabular}{|c|c|c|c|c|c|}
\hline Durée & & & $35 \mathbf{j}$ & $42 j$ & $26 \mathrm{j}$ \\
\hline Dates & 16.5 & 12.6 & 16.7 & 28.8 & 12.9 \\
\hline $\mathrm{N}$ & \multicolumn{2}{|c|}{11} & 11 & 11 & 11 \\
\hline $\begin{array}{l}\text { Croissance journalière } \\
\text { moyenne }(\mathrm{g})\end{array}$ & \multicolumn{2}{|c|}{303,03} & 374,02 & 547,61 & 395,10 \\
\hline \multirow[t]{3}{*}{ Croissance } & \multicolumn{3}{|c|}{343,10 (pendant 62 j) } & & \\
\hline & \multicolumn{3}{|c|}{425,69 (pendant 104 j) } & & \\
\hline & \multicolumn{4}{|c|}{419,58 (pendant $130 \mathrm{j}$ ) } & \\
\hline
\end{tabular}


Le croît a subi de fortes variations selon les semaines d'observation, et le comportement individuel a été très variable.

Cinq animaux, soit 38 p. 100 de l'effectif, ont dépassé les $50 \mathrm{~kg}$ de gain.

Le record se situe à $115 \mathrm{~kg}$ de croît, ce qui représente un gain quotidien de $891 \mathrm{~g}$.

Le gain quotidien moyen a augmenté pendant les trois premiers mois et diminué ensuite.

Les meilleurs résultats ont été obtenus entre le 10 et le 24 juillet après la période de perte de poids due à l'inconfort de la litière. Les animaux ont alors gagné $1.298 \mathrm{~g}$ pendant les sept premiers jours et $1.025 \mathrm{~g}$ pendant les sept derniers.

Ces performances n'ont été obtenues que pendant une courte durée et peuvent être attribuées en partie au croît compensateur.

\section{Consommations}

Panicum maximum: Le niveau de consommation du Panicum s'est trouvé d'emblée très élevé. Les variations hebdomadaires sont toutefois très importantes, les quantités consommées variant de 16 à $26 \mathrm{~kg}$ par animal et par jour. La moyenne journalière s'établit à $18 \mathrm{~kg}$ par animal, ce qui représente une consommation de $3,4 \mathrm{~kg}$ de matières sèches pour 1,98 UF et $261 \mathrm{~g}$ de matières azotées digestibles.

Farines de riz: Les quantités de farines de riz consommées quotidiennement s'élèvent régulièrement. Elles passent de 2 à $5,1 \mathrm{~kg}$ par animal.

L'on a observé cependant des variations notables d'une semaine à l'autre et la moyenne des consommations a été de $3,8 \mathrm{~kg}$ par animal.

TABLEAU $N^{\circ}$ III

Consomnation

\begin{tabular}{|c|c|c|c|c|}
\hline & & Matières sẽches & U.F. & M.A.D. \\
\hline \multirow{3}{*}{$\begin{array}{l}\text { Total du lot } \\
\text { en } 130 \text { jours }\end{array}$} & Panicum & $5579 \mathrm{~kg}$ & 3068 & $390 \mathrm{~kg}$ \\
\hline & Farines de riz & $4557 \mathrm{~kg}$ & 4.557 & $\underline{509 \mathrm{~kg}}$ \\
\hline & Total & $10131 \mathrm{~kg}$ & 7625 & $999 \mathrm{~kg}$ \\
\hline $\begin{array}{l}\text { Moyenne par } \\
\text { animal et par } \\
\text { jour }\end{array}$ & & $6,54 \mathrm{~kg}$ & 5,2 & $623 \mathrm{~g}$ \\
\hline
\end{tabular}

\section{Abreuvement}

La consommation d'eau a été très irrégulière. Elle a varié de 12,6 litres à 29,5 litres par animal et par jour et la consommation moyenne s'est établie à 18,8 litres par animal et par jour.

\section{Indices de consommation}

L'indice de consommation a varié selon les périodes.

La valeur de l'indice cumulé pour l'ensemble de l'expérience est de 12,9. Cette valeur

TABLEAU N*IV

Indices de consommation

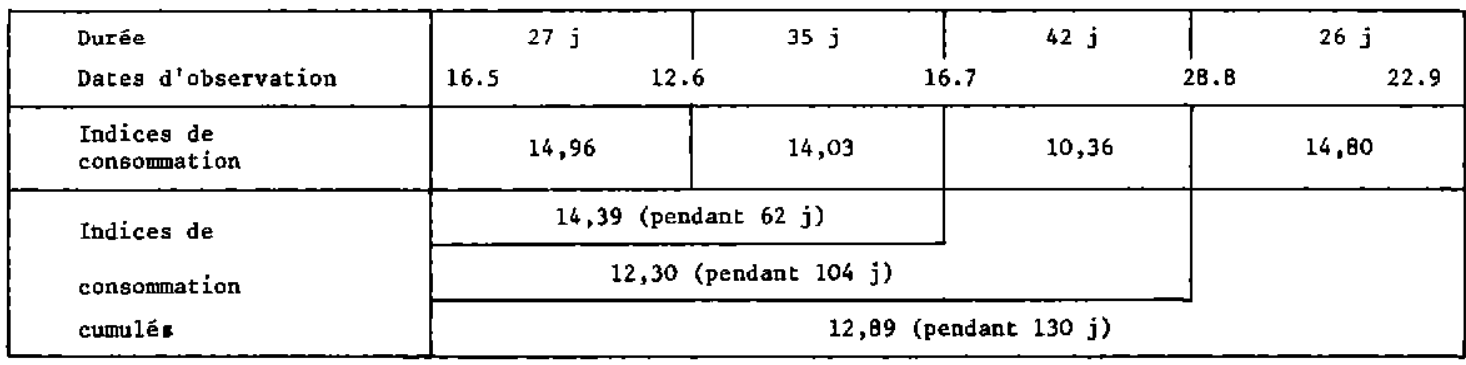


relativement élevée de l'indice indique que la croissance obtenue est surtout réalisée à base de graisse et non de viande, ce qui a été confirmé à l'étude des carcasses.

L'économie des gains attendus s'en ressent d'autant. Notons toutefois que la valeur de 12,9 est nettement inférieure à celle de la pré- cédente expérience où l'indice de consommation était de 19.

\section{Etude des carcasses}

Les animaux ont été abattus en un seul lot, le 23 septembre à l'abattoir d'Abidjan.

TABLEAUU $\mathbb{N}^{\circ} \mathrm{V}$

Etude des carcasses

\begin{tabular}{|l|c|c|c|c|}
\hline & Lot d'expérience & Témoins & Classe I & Classe I \\
\hline Poids vif & 283,4 & 236,5 & 323 & 301 \\
Poids de carcasse & 155,4 & 127,5 & 190 & 175 \\
Rendement sur poids vif & 54,83 & 52,69 & 58,82 & 58,13 \\
Longueur de la carcasse & 111 & 110 & 118 & 111 \\
Epaisseur de la cuisse & 19,5 & 15,7 & 20,0 & 3,5 \\
Epaisseur du plat de côte & 2,7 & 2,0 & 10,52 & 3,0 \\
Epaisseur de la cuisse en & 12,58 & 11,0 & 16,94 & 12,40 \\
p.loo du poids de carcasse & 17,56 & 14,2 & & 19,54 \\
Epaisseur de 1a cuisse en & & & & \\
p.100 de la longueur & & & & \\
\hline
\end{tabular}

La mesure de l'épaisseur de la cuisse, en p.100 du poids de la carcasse et en p.100 de la longueur, dont la valeur est élevêe estíl'indice d'une bonne conformation bouchère et rêvèle un pourcentage élevê des morceaux de première catégorie.

Dans le tableau V sont représentées les moyennes des observations effectuées sur les animaux d'expérience, les moyennes des mesures faites sur les témoins au début de l'expérience et les résultats obtenus sur le meilleur zébu du lot expérimental (Classe I).

Pour l'ensemble du lot la conformation bouchère est bonne, le gras de carcasse est abondant et les rognons bien couverts, sans fenêtre. La graisse de couverture est importante.

Le rendement de carcasse sur poids vif est variable selon les animaux, avec des valeurs allant de 50,19 à 58,83 p. 100 ; la valeur moyenne est de 54,83 p. 100 . La valeur du rendement est nettement améliorée par rapport à la valeur observée sur les animaux témoins abattus au début de l'expérience.

\section{BILAN ECONOMIQUE}

L'aspect économique de pareilles opérations est extrêmement important pour le développement des entreprises d'embouche.
Au cours de ces deux expériences d'embouche intensive, un certain nombre d'informations ont été rassemblées qui permettent de calculer les prix de revient et d'évaluer la possible rentabilité de ces opérations.

La lecture de ces tableaux permet de tirer les conclusions générales suivantes :

1. Dans les conditions de l'essai, la valeur ajoutée par l'embouche ne permet pas de couvrir les frais de production et d'assurer la rentabilité des opérations effectuées selon le modèle technique proposé. Cela est dû :

- à la faiblesse des gains de poids obtenus;

- à la lourdeur des charges en raison du coût élevé des rations alimentaires et de l'importance des risques zootechniques et sanitaires.

- à la valeur réduite de la marge bénéficiaire attendue en raison du prix d'achat élevé du maigre et de l'absence de plus-value à la qualité.

2. Dans la mesure où la mise au point d'un système d'entretien économique permettrait de 
réduire les coûts de production, une stratégie des prix fixant le prix d'achat des animaux maigres et prévoyant une valorisation à 125 ou $130 \mathrm{~F} \mathrm{le} \mathrm{kg}$ pour les animaux de qualité embouche permettrait une rentabilité plus aisée des opérations d'engraissement intensif en diminuant la rigueur des impératifs zootechniques et en relevant la marge bénéficiaire.

3. Une telle politique constituerait une remarquable incitation à la production intensive d'animaux d'embouche, ce qui permettrait à la Côte d'Ivoire d'aménager des emplois nouveaux dans le secteur primaire et de limiter dans un délai relativement court son déficit

TABLEAU $\mathrm{N}^{\circ} \mathrm{VI}$

Coût global des opérations d'embouche

\begin{tabular}{|lll|}
\hline Expérience I : & Après 2 mois & 6024 \\
\hline & Après 3 mois & 7761 \\
\hline Après 4 mois & 9559 \\
\hline Expérience II : & Après 2 mois & 5960 \\
\hline & Après 3 mois & 7896 \\
\hline & Après 4 mois & 9806 \\
\hline
\end{tabular}
en viande.

abadue definissant le seuIL de rentabilite des operations d'embouche

Gain de poids $\mathrm{kg}$

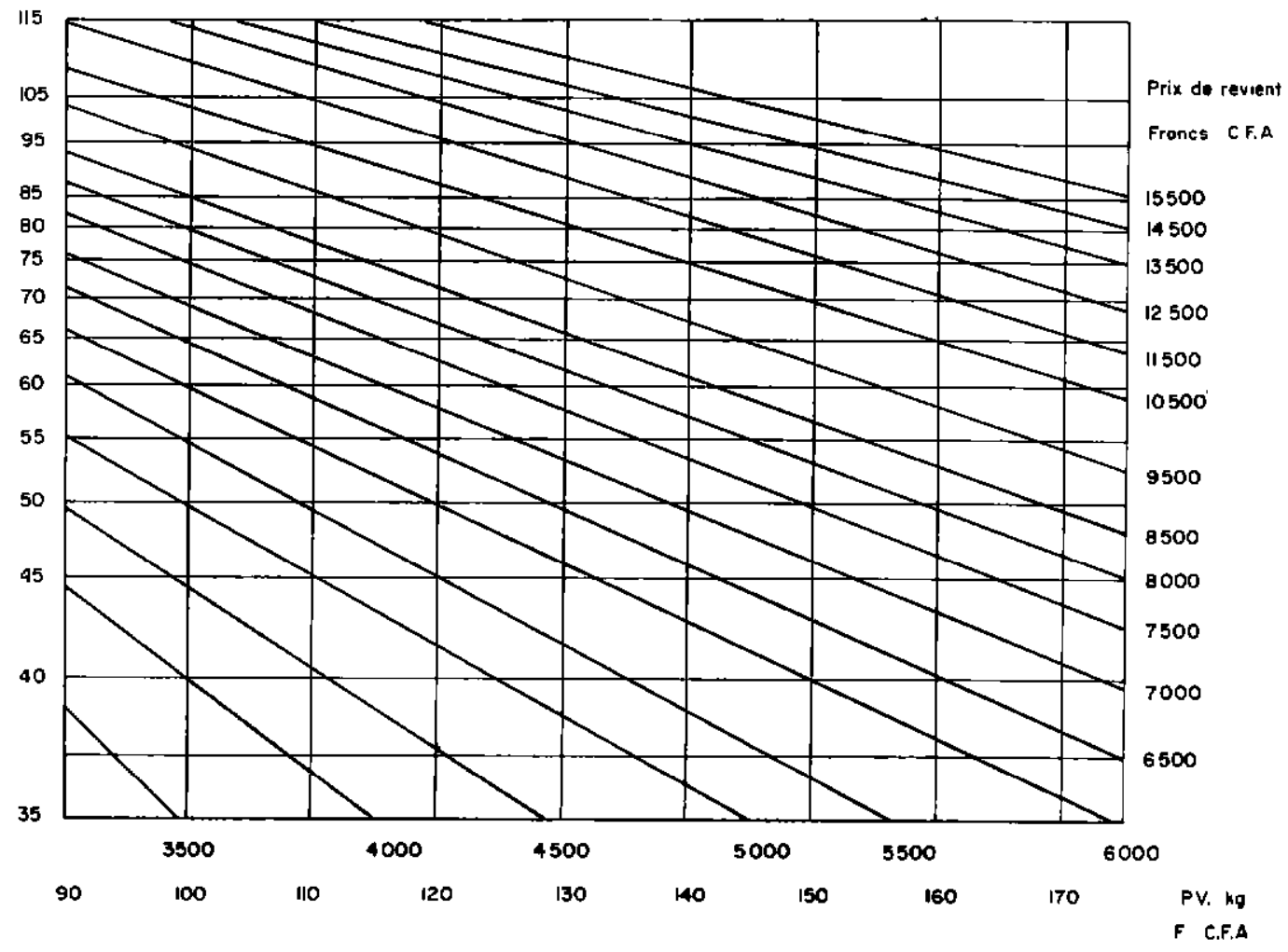

\section{CONCLUSIONS}

L'embouche intensive en parc de stabulation permanente avec affouragement à l'auge est une méthode éprouvée, ayant donné, en particulier au Sénégal, des gains de poids de plus d'un $\mathrm{kg}$ par jour sur des zébus saheliens de race Gobra.
L'application immédiate de cette méthode en Côte d'Ivoire est plus aléatoire dans la mesure où :

1. Le matériel animal disponible sur place, (il s'agit de zébus maliens importés) ne réagit 
pas aussi favorablement à l'embouche en parc que le laissaient présumer les expériences réalisées à Dakar ou à Madagascar par exemple.

2. Les charges de structure relatives à la réalisation du modèle technique proposé sont d'autant plus lourdes que les marges bénéficiaires attendues sont limitées par le prix d'achat élevé des animaux maigres et l'impossibilité actuelle d'obtenir la valorisation des carcasses de cette qualité.

Tous ces facteurs limitent le développement des entreprises d'embouche, dont la mise en place à court terme est cependant indispensable à la Côte d'Ivoire, si ce pays souhaite limiter la valeur de son déficit en viande bovine.
Dans ces conditions il conviendrait:

- de reconsidérer, pour la Côte d'Ivoire, le modèle technique proposé en étudiant l'économie de l'embouche des zébus sur pâturage artificiel permanent, complémenté.

- d'envisager le développement de l'embouche intensive sur d'autres types d'animaux présentés en Côte d'Troire, animaux de race N'Dama, Baoulé, animaux métis issus de croisement Jersey-N'Dama.

- qu'intervienne sur le plan commercial un aménagement du prix de la viande de cette qualité, ne serait ce que pour créer une incitation à la production intensive de viande bovine, actuellement négligée en Côte d'Ivoire.

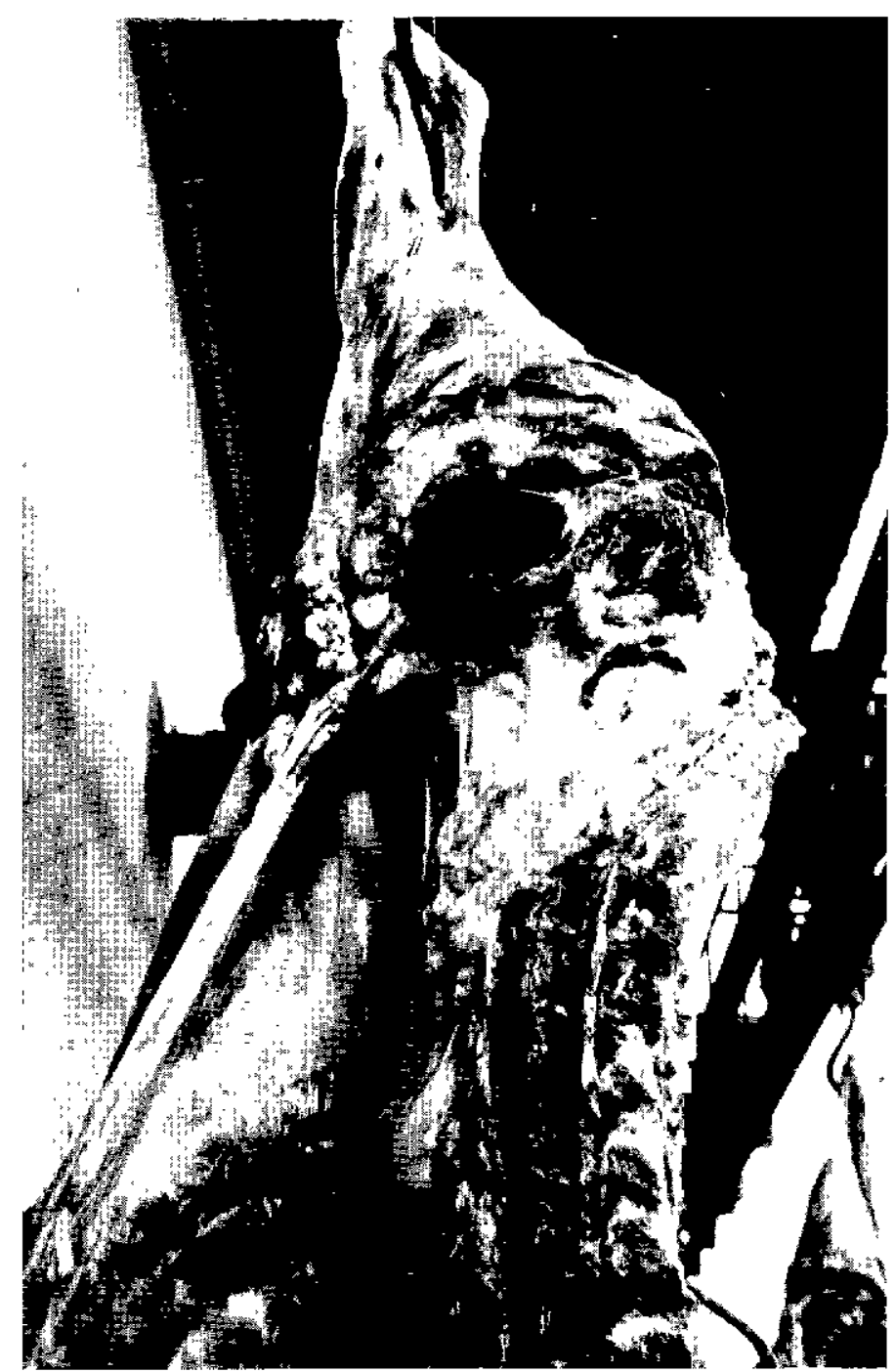

Carcasse de zébu malien. Pan. 


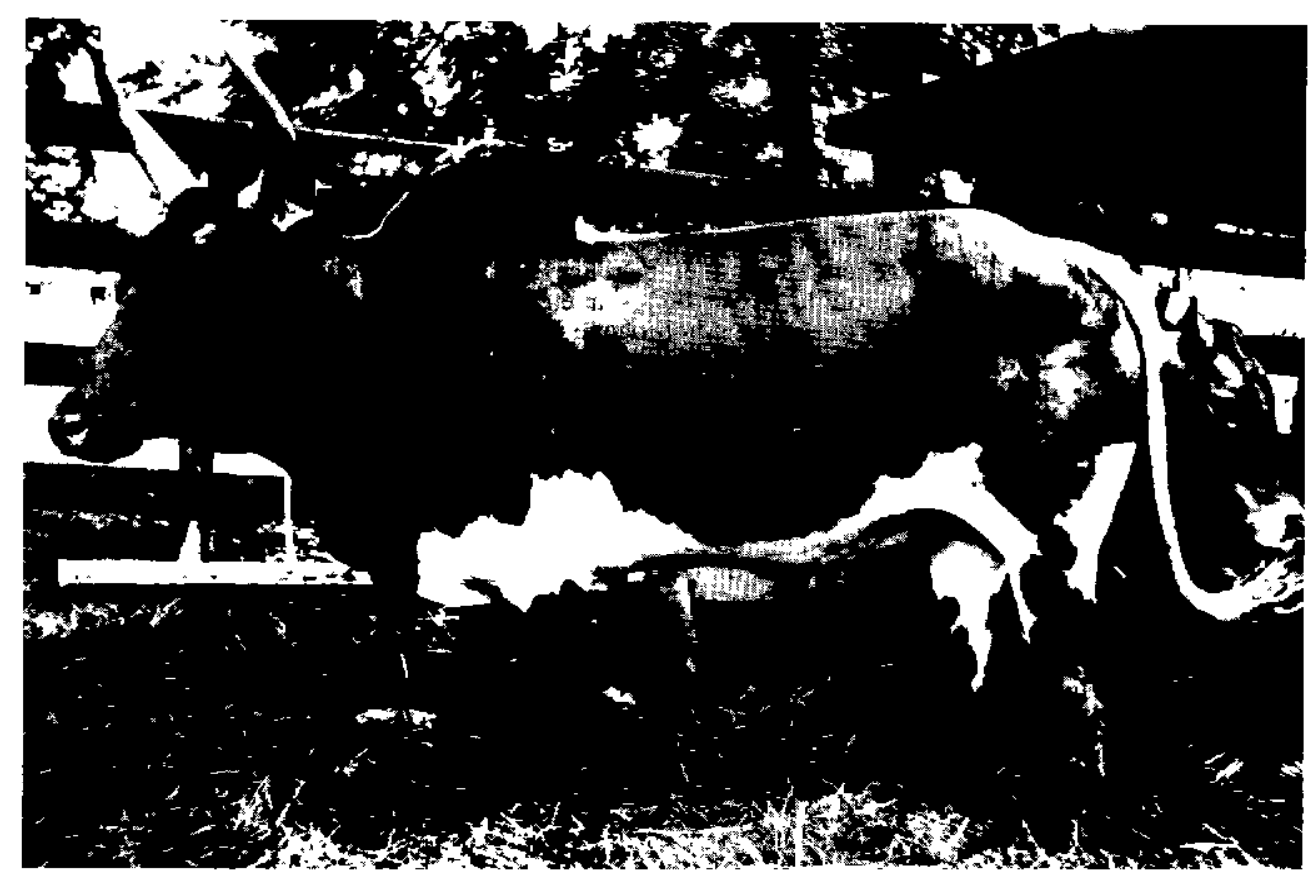

Zébus maliens après l'embouche.

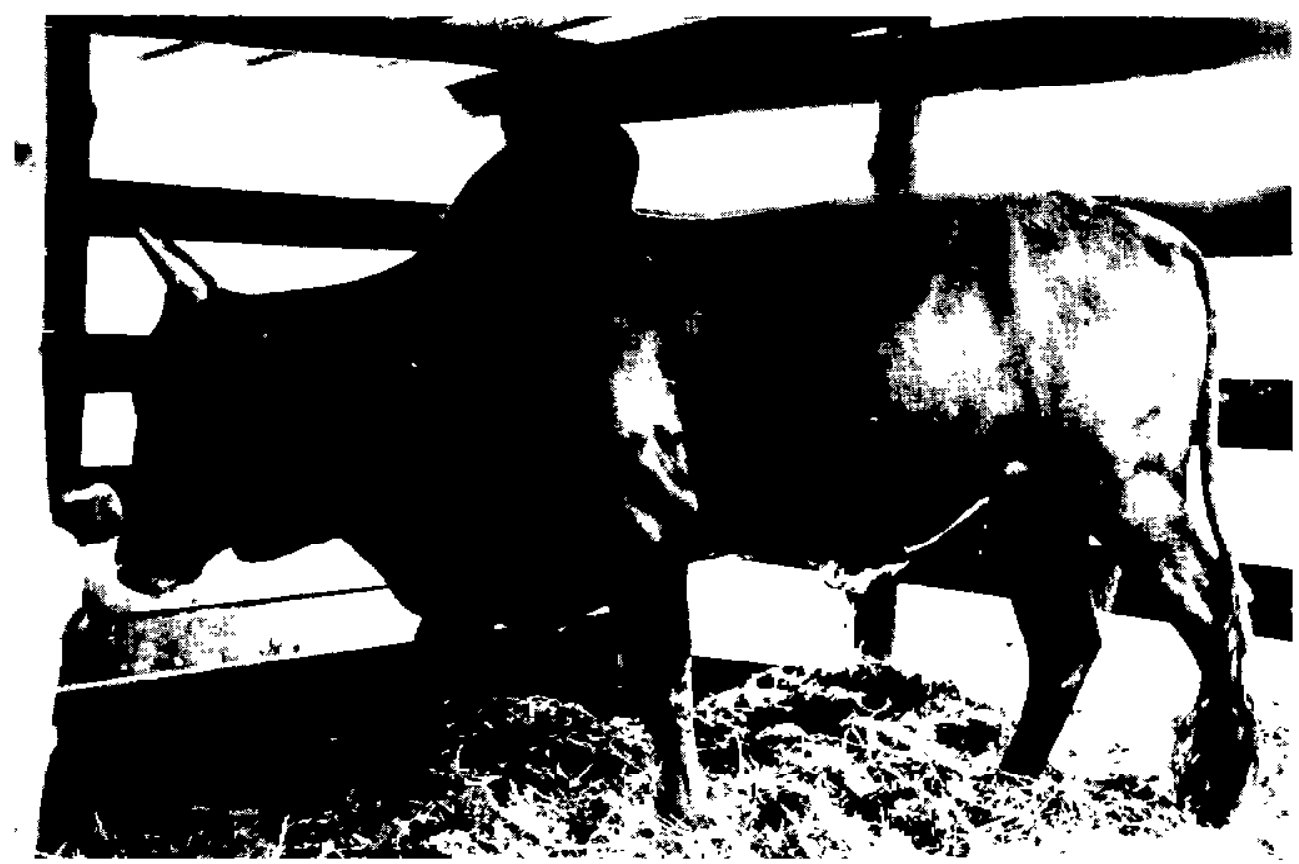




\section{RESUME}

Dans une première expérience, des zébus maliens, âgés de 6 à 9 ans, castrés, pesant en moyenne au départ $331 \mathrm{~kg}$ ont reçu pendant 138 jours une alimentation intensive à base de Stylosanthes gracilis et de farines basses de riz, avec un gain moyen journalier de $298 \mathrm{~g}$ environ pour un indice de consommation d'ensemble de 19 U.F.

Ces résultats, nettement médiocres, sont à mettre pour partie au compte de l'hétérogénéité du lot et de l'âge des animaux, car dans une deuxième expérience où le Stylosanthes a été remplacé par du Panicum maximum portant sur un lot plus homogène d'animaux, âgés de 3 à 5 ans, castrés, d'un poids moyen de $231 \mathrm{~kg}$, ce gain a atteint $420 \mathrm{~g}$ pour un indice de consommation de 12,8 U.F. Ces résultats sont cependant trop faibles pour justifier localement, eu égard aux charges, la vulgarisation de cette technique.

\section{SUMMARY}

\section{Intensive fattening experiments of Mali zebu cattle}

In a first experiment 6 to 9 years old castrated Mali zebu cattle weighing on an average $331 \mathrm{~kg}$ were fed intensively during 138 days with Stylosanthes gracilis and rice polishings. A daily average gain of about $298 \mathrm{~g}$ with a food conversion efficiency of 19 F.U. was observed.

These quite feeble results are partly to put in down to the heterogeneity of the lot and to the age of the animals; because in a second experiment in which a lot of more homogeneous 3 to 5 years old castrated cattle weighing on an average $231 \mathrm{~kg}$, the daily live-weight gain was $420 \mathrm{~g}$ with a food conversion efficiency of $12,8 \mathrm{~F}$.U.

Yet, these results are too feeble to warrant locally, in consideration of charges, the vulgarization of this technics.

\section{RESUMEN}

\section{Ensayos de engorde intensivo de cebues de Mali}

En la primera experiencia, durante 138 dias se alimentaron intensivamente con Stylosanthes gracilis y harinillas de arroz cebues de Mali, de 6 a 9 años de edad, castrados, de un peso medio de $331 \mathrm{~kg}$ al principio. El aumento medio diario de peso llegó a unos $298 \mathrm{~g}$ con indice de consumo de conjunto de 19 unidades nutritivas.

Dichos resultados terminantemente mediocres provienen por parte de la heterogeneidad del lote y de la edad de los animales, porque, en una segunda experiencia en que se utilizó Panicum maximum en lugar de Stylosanthes para alimentar un lote más homogéneo de animales de 3 à 5 años de edad, castrados, de un peso medio de $231 \mathrm{~kg}$, fué de $420 \mathrm{~g}$ el aumento de peso con un indice de consumo de 12,8 U.N. Sin embargo, dichos resultados son demasiado medianos para justificar localmente la vulgarización de esta técnica, en consideración de las cargas. 